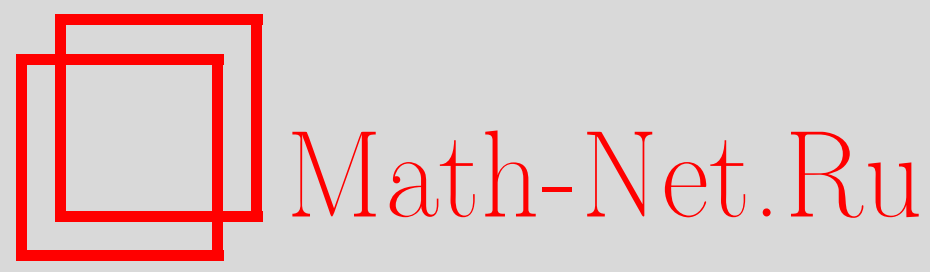

Б. З. Шаваровский, Редукция матриц при помощи эквивалентных и подобных преобразований, Матем. заметки, 1998, том 64, выпуск 5, 769-782

DOI: https://doi.org/10.4213/mzm1453

Использование Общероссийского математического портала Math-Net.Ru подразумевает, что вы прочитали и согласны с пользовательским соглашением http://www.mathnet.ru/rus/agreement

Параметры загрузки:

IP : 3.91 .87 .62

26 апреля 2023 г., 16:44:57

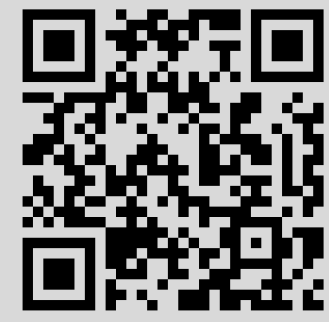




\section{РЕДУКЦИЯ МАТРИЦ ПРИ ПОМОЩИ ЭКВИВАЛЕНТНЫХ И ПОДОБНЫХ ПРЕОБРАЗОВАНИЙ}

\section{Б. З. Шаваровский}

Исследуется структура многочленных матриц в связи с приводимостью их полускалярно эквивалентными и подобными преобразованиями к более простым видам. В частности, указывается каноническая форма многочленных матриц без кратных характеристических корней относительно указанных преобразований. Это позволяет установить каноническую форму относительно подобия для одного типа конечных наборов числовых матриц.

Библиографоия: 13 названий.

1. Введение. В целом настоящая статья посвящена исследованию структуры матриц над полями и кольцами многочленов с точки зрения их приводимости различньми преобразованиями к более простым видам. В этом направлении имеется обширная литература. Отметим, например, работы [1]-[5].

В 1976 году П. С. Казимирский и В. М. Петричкович наряду с известньми понятиями эквивалентности и скалярной (строгой) эквивалентности [6], [7] ввели понятие полускалярной эквивалентности многочленных матриц [8], [9]. Их обшими усилиями установлена некоторая простая форма (треугольная с инвариантными множителями на главной диагонали), к которой приводится матрища посредством полускалярно эквивалентных преобразований (см. также [5]). Однако матрица указанной формы определяется неоднозначно, причем неопределенность здесь слишком велика. Следовательно, для решения задачи классификации матриц относительно полускалярной эквивалентности необходимо доопределить установленную треугольную форму (или построить новую) так, чтобы она была единственной с точностью до некоторого тривиального различия. В общем случае это очень сложная задача, напрямую связанная с классической проблемой линейной алгебры о приведении к каноническому виду пары числовых матриц одновременным преобразованием подобия (решение этой проблемы см. в [4]). Поэтому естественно сузить класс многочленных матрищ настолько, чтобы эта задача уже поддавалась решению.

Объектом исследования в данной работе являются квадратные неособенные многочленные матрицы без кратных характеристических корней, а основная цель - приведение таких матриц полускалярно эквивалентными преобразованиями к каноническому виду. При этом вводится понятие обобщенных элементарных делителей, которые при переходе к многочленным матрицам первой степени совпадают с обычньпи элементарными делителями. Далее, применяя полученные результаты, решается задача о канонической форме относительно одновременного преобразования подобия конечных наборов 
числовых матриц при том же предположении об отсутствии кратных характеристических корней у сопоставляемых наборам многочленных матриц. Предлагаемая здесь каноническая форма является набором матриц одинаковой квазидиагональной структуры, причем каждый из наборов соответствующих диагональных блоков в свою очередь является набором матриц одинакового квазитреугольного строения. Обратим внимание, что метод построения указанной формы в классе подобных всегда дает единственньй с точностью до одновременной перестановки прямых слагаемых набор матриц с максимально возможным числом диагональных блоков, а характеристические многочлены диагональных блоков многочленной матрицы, сопоставляемой каноническому набору матриц, совпадают с его обобщенными элементарными делителями. Важно отметить, что в случае, когда набор состоит из одной лишь матрицы, построенная здесь каноническая форма совпадает в точности с канонической формой Жордана.

Для сравнения приведем смежные работы [10], [11], в которых указьваются условия полускалярной эквивалентности отдельных классов матрищ.

Отметим, наконец, что методполускалярной эквивалентности сыграл важную роль в построении Казимирским теории разложения матричных многочленов на множители [2] (в этом направлении см. также монографию [3]).

2. Определения и вспомогательные предложения. Основным полем здесь и в дальнейшем будет линейно упорядоченное лексикографическим способом поле комплексных чисел $\mathbb{C}$.

ОПРедЕЛЕниЕ 1. Прямоугольные матрищы $A$ и $A^{\prime}$ называются диагонально әквивалентными (в обозначениях $A \stackrel{d}{\sim} A^{\prime}$ ), если $A^{\prime}=B A C$, где $B$ и $C$ - диагональные неособенные матрицы [12].

ОПРЕДЕЛЕНИЕ 2. Строки $\bar{a}_{1}$ и $\bar{a}_{2}$ одинаковой длины называются связанными (обобщение аналогичного понятия из работы [10]), если существует номер $i$ такой, что $i$-е элементы в этих строках одновременно отличны от 0 . Система строк $\left(\bar{a}_{1}, \ldots, \bar{a}_{s}\right)$ называется связанной, если существует их перестановка $\left(\bar{a}_{i_{1}}, \ldots, \bar{a}_{i_{s}}\right)$ такая, что каждая последующая строка связана с некоторой из предыдущих. Две матрищы $A$ и $B$ называются связанными, если некоторая строка одной из них связана с некоторой строкой другой.

Нетрудно проверяется следующее утверждение.

ПРЕДЛОЖЕНИЕ 1. Матрииа со связанной системой ненулевых строк и более чем одним ненулевым столбцом перестановками строк и столбцов приводится $\kappa в u \partial y$

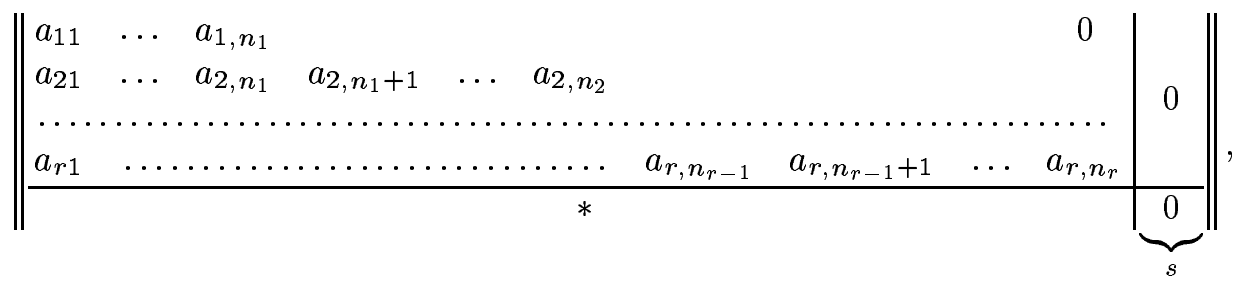

әде все әлементы $a_{11}, \ldots, a_{1, n_{1}}, a_{2, n_{1}+1}, \ldots, a_{2, n_{2}}, \ldots, a_{r, n_{r-1}+1}, \ldots, a_{r, n_{r}}$ и все подстроки $\left\|a_{21} \ldots a_{2, n_{1}}\right\|, \ldots,\left\|a_{r 1} \ldots a_{r, n_{r-1}}\right\|$ ненулевые, $1<n_{1}<\ldots<n_{r}, s \geqslant 0$. 
Для определения канонической формы многочленной матрицы в классе полускалярно эквивалентных введем понятие псевдостохастической матрищы и решим вопрос о построении в классепсевдостохастических диагонально эквивалентных матриц некоторой стандартной формы.

ОПРЕДЕЛЕниЕ 3. Числовая матрища назьвается псевдостохастической, если сумма элементов каждой ее строки равна единице.

Очевидно, диагонально эквивалентные преобразования, вообще говоря, не сохраняют свойство псевдостохастичности. Поэтому к псевдостохастическим матрицам будем применять только те преобразования, которые не нарушают это свойство.

Под позицией некоторого элемента матрищы, как обычно, будем понимать пару чисел $(i, j)$, где $i$ и $j$ - номера строки и столбца соответственно, в которых находится этот элемент.

Отбросив тривиальньй случай, когда каждая строка матришы содержит лишь один ненулевой элемент, рассмотрим псевдостохастическую матрицу $A$, система строк которой связанная. Согласно предложению 1 перестановками строк и столбцов она приводится к виду (1). Позиции матрицы $A$, которые занимают ненулевые элементы $a_{11}, \ldots$, $a_{1, n_{1}-1}, a_{2, n_{1}+1}, \ldots, a_{2, n_{2}-1}\left(\right.$ если $\left.n_{2}-n_{1}>1\right), \ldots, a_{r, n_{r-1}+1}, \ldots, a_{r, n_{r}-1}\left(\right.$ если $n_{r}-$ $\left.n_{r-1}>1\right), a_{2, s_{1}}\left(1 \leqslant s_{1} \leqslant n_{1}\right), \ldots, a_{2, s_{r-1}}\left(1 \leqslant s_{r-1} \leqslant n_{r-1}\right)$ матрицы (1), назовем определяющими позиииями матрицы $A$. Понятно, что матрица $A$ может иметь несколько множеств определяющих позиций, поскольку матрица (1) определяется неоднозначно. Упорядочим каждое из этих множеств лексикографическим способом. Полученные таким образом упорядоченные множества назовем наборами определяющих позииий (НОП) данной матрицы. Пусть, далее, $\mathscr{P}=\left\{\left(i_{1}, j_{1}\right),\left(i_{2}, j_{2}\right), \ldots,\left(i_{p}, j_{p}\right)\right\}$, $\mathscr{P}^{\prime}=\left\{\left(i_{1}^{\prime}, j_{1}^{\prime}\right),\left(i_{2}^{\prime}, j_{2}^{\prime}\right), \ldots,\left(i_{q}^{\prime}, j_{q}^{\prime}\right)\right\}-$ два НОП матрицы $A$. Будем считать, что $\mathscr{P}<\mathscr{P}^{\prime}$ $(\mathscr{P}$ “меньше" $\mathscr{P})$, если $\left(i_{1}, j_{1}\right)=\left(i_{1}^{\prime}, j_{1}^{\prime}\right), \ldots,\left(i_{u}, j_{u}\right)=\left(i_{u}^{\prime}, j_{u}^{\prime}\right)$, но $\left(i_{u+1}, j_{u+1}\right)<$ $\left(i_{u+1}^{\prime}, j_{u+1}^{\prime}\right), 0 \leqslant u<\min (p, q)$. Среди всех НОП матрищы $A$ можем найти единственньй набор $\mathscr{P}$ такой, что $\mathscr{P}<\mathscr{P}^{\prime}$ для каждого НОП $\mathscr{P}^{\prime} \neq \mathscr{P}$. Назовем его первым набором определяющих позичий (ПНОП) матрицы $A$. Поскольку под действием диагонально эквивалентных преобразований позиции ненулевых элементов матрищы остаются неизменными, то ПНОП является характеристикой не одной матрицы $A$, а целого класса $\{B A C\}$ диагонально эквивалентных матриц. Каждой матрице $D \in\{B A C\}$ соответствует набор $K_{D}$ ее элементов, стоящих в ПНОП. Это соответствие взаимно однозначно. Классу $\{B A C\}$ соответствует множество $K=\left\{K_{D}\right\}$ наборов элементов из $\mathbb{C}$. Предполагая основное поле комплексных чисел линейно упорядоченным лексикографическим способом, можем упорядочить теперь множество $K$ также лексикографическим способом. Если множество $\mathscr{L}$ матриц $G \in\{B A C\}$ таких, что $K_{G}$ является набором натуральных чисел, непусто, можем выбрать матрицу $H \in \mathscr{L}$ с минимальным $K_{H}$. Назовем ее канонической в классе псевдостохастических диагонально эквивалентных матриц.

ЗАмЕчАниЕ. Название "каноническая" здесь несколько условное, поскольку диагонально эквивалентные преобразования отнюдь не упрощают матрицу в смысле увеличения числа ее нулевых элементов и придания ей более простого вида.

ПРЕДЛОЖЕНИЕ 2. В классе $\{B A C\}$ псевдостохастических диагонально эквива- 
лентных матриц, со связанной системой строк существует, притом единственная, каноническая матрича.

ДокАЗАТЕЛЬСтво. Сушествование. Наряду с данной псевдостохастической матрицей $A$ построим матрицу $Z$ тех же размеров, с тем же размещением нулевых элементов и с неопределенными остальными ненулевыми элементами. Пусть $\left(z_{i_{1}}, \ldots, z_{i_{k}}\right)$ - набор элементов матрицы $Z$, стоящих в ПНОП матрицы $A$. Будем считать, что построенная матрица псевдостохастическая и все отношения произведений нормальных наборов (ненулевых) элементов [6] каждой ее квадратной подматрицы равны соответствующим отношениям для матрицы $A$. Следовательно, неизвестные элементы матрицы $Z$ являются зависимыми. Нетрудно показать, что каждьй ее ненулевой элемент имеет вид $z_{i}=u_{i} / v_{i}$, где $u_{i}, v_{i} \in \mathbb{C}\left[z_{i_{1}}, \ldots, z_{i_{k}}\right]$. Придавая переменным $z_{i_{1}}, \ldots, z_{i_{k}}$ допустимые значения такие, что $u_{i} \neq 0, v_{i} \neq 0$, получаем конкретные (из основного поля) значения $z_{i}$. Во вполне упорядоченном (лексикографическим способом) непустом множестве наборов $\left\{\left(a_{1}, \ldots, a_{k}\right)\right\}, a_{j} \in \mathbb{N}$, допустимых для набора неизвестных $\left(z_{i_{1}}, \ldots, z_{i_{k}}\right)$, выбираем первьй (минимальньй) набор $\left(a_{10}, \ldots, a_{k 0}\right)$. Заменив все те элементы матрицы $Z$, которые являются единственньми ненулевыми элементами своих строк, единицами и набор $\left(z_{i_{1}}, \ldots, z_{i_{k}}\right)$, элементов из ПНОП набором $\left(a_{10}, \ldots, a_{k 0}\right)$, вычислив при этом все остальные элементы $z_{i}=u_{i} / v_{i}$, получим числовую псевдостохастическую каноническую матрицу $Z_{0}$, которая согласно [12] диагонально эквивалентна матрице $A$.

Единственность достаточно доказать для случая, когда матрища не содержит нулевых столбцов. Пусть имеем две канонические $(k \times t)$-матрицы $M$ и $M^{\prime}$ с одинаковьп размещением нулевых элементов и равньми соответствующими элементами в ПНОП. Не уменьшая общности, будем считать, что все позиции из ПНОП в этих матрицах находятся в первых $r$ строках $(r \geqslant 1)$. На основании [12] из условия

$$
\operatorname{diag}\left(\alpha_{1}, \ldots, \alpha_{k}\right) M=M^{\prime} \operatorname{diag}\left(\beta_{1}, \ldots, \beta_{t}\right)
$$

где все $\alpha_{i}, \beta_{j}$ ненулевые, вытекает, что первые $r$ строк матрицы $M$ совпадают с соответствующими строками матрицы $M^{\prime}$. Таким образом, $\alpha_{1}=\cdots=\alpha_{r}=\beta_{1}=\cdots=\beta_{t}$. Пусть, далее, $\bar{a}_{u}$ и $\bar{a}_{u}^{\prime}-u$-е строки матриц $M$ и $M^{\prime}$ соответственно, причем $u>r$. Тогда согласно доказанному выше из условия (2) имеем $\alpha_{u} \bar{a}_{u}=\alpha_{1} \bar{a}_{u}^{\prime}$. Но поскольку сумма элементов каждой из строк $\bar{a}_{u}, \bar{a}_{u}^{\prime}$ равна 1 , то $\alpha_{1}=\alpha_{u}$; следовательно, $M=M^{\prime}$.

3. Полускалярная эквивалентность многочленных матриц без кратных характеристических корней. Рассмотрим кольцо $\mathbb{C}_{n}[x](n \times n)$-матриц над $\mathbb{C}[x]$.

ОПРЕДЕЛЕНИЕ 4. Матрищы $A(x), B(x) \in \mathbb{C}_{n}[x]$ называются полускалярно әквивалентными (в обозначениях $A(x) \sim B(x)$ ), если $B(x)=C A(x) Q(x)$ для некоторых $C \in \mathrm{GL}_{n}(\mathbb{C}), Q(x) \in \mathrm{GL}_{n}(\mathbb{C}[x])[2],[8]$.

Наша задача - установить каноническую форму в классе полускалярно эквивалентных квадратных многочленных матриц без кратных характеристических корней.

Рассмотрим произвольную матрицу $A(x) \in \mathbb{C}_{n}[x]$, все корни характеристического многочлена $\Delta(x)=\operatorname{det} A(x)$ которой имеют кратность 1. Договоримся в дальнейшем значение произвольной матрицы $G(x)$ на системе корней многочлена $\varphi(x)$ (см. [2, определение $1, \S 2$, разд. 11], обозначение $\left.M_{G(x)}(\varphi)\right)$ записьвать в порядке возрастания корней, т.е. если, например, многочлен $\varphi(x)$ имеет все простые корни $\alpha_{1}, \ldots, \alpha_{s}$, причем 
$\alpha_{i_{1}}<\cdots<\alpha_{i_{s}}$, то

$$
M_{G(x)}(\varphi)=\left\|\begin{array}{c}
G\left(\alpha_{i_{1}}\right) \\
\cdots \\
G\left(a_{i_{s}}\right)
\end{array}\right\|
$$

ПРЕДЛОЖЕНИЕ 3. Матрица $A(x)$ полускалярно әквивалентна матрище вида

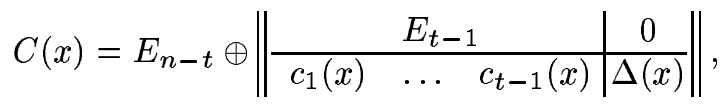

әде $E_{m}-$ единичная матрица порядка $m, t=\operatorname{rank} M_{A_{*}(x)}(\Delta)\left(A_{*}(x)\right.$ - взаимная матрица для $A(x))$ и для произвольно фиксированных характеристических корней $\alpha_{1}, \ldots, \alpha_{t}$ таких, что $\operatorname{rank} M_{A_{*}(x)}\left[\alpha_{1}, \ldots, \alpha_{t}\right]=t$, выполняются условия

$$
c_{i}\left(\alpha_{j}\right)=\delta_{i j}, \quad i, j=1, \ldots, t, \quad c_{t}(x)=1-\sum_{k=1}^{t-1} c_{k}(x)
$$

где $\delta_{i j}-$ символ Кронекера.

ДокАЗАТЕЛЬСТво. Если $\operatorname{rank} M_{A_{*}(x)}(\Delta)=t$, но на основании теоремы 1 из [9] и предложений 3,4 из $\left[2, \S 2\right.$, разд. 11] можно показать, что $A(x) \sim E_{n-t} \oplus B(x)$, где $B(x)$ имеет вид

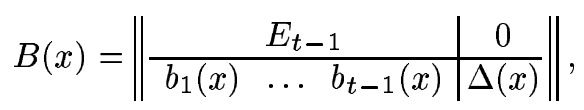

причем $\operatorname{rank} M_{B_{*}(x)}(\Delta)=t, \operatorname{deg} b_{r}<\operatorname{deg} \Delta, r=1, \ldots, t-1$. В случае $t=1$ уже все доказано. Пусть $t>1$ и для некоторых характеристических корней $\alpha_{1}, \ldots, \alpha_{t}$ вьполняется условие $\operatorname{rank} M_{A_{*}(x)}\left[\alpha_{1}, \ldots, \alpha_{t}\right]=t$. Складывая первые $t-1$ строк матрищы $B(x)$, умноженные на некоторые константы, с последней ее строкой, получим матрицу вида (5), последняя строка которой $\left\|b_{1}^{(0)}(x) \ldots b_{t-1}^{(0)}(x) \Delta(x)\right\|$, где $b_{r}^{(0)}\left(\alpha_{t}\right)=0, r=1, \ldots, t-1$. Тогда $\left\|b_{1}^{(0)}\left(\alpha_{1}\right) \ldots b_{t-1}^{(0)}\left(\alpha_{1}\right)\right\| \neq \overline{0}$, и мы можем считать, что $b_{1}^{(0)}\left(\alpha_{1}\right)=1$. Далее, прибавляя некоторые кратные первого столбца ко 2 -му $, \ldots,(t-1)$-му столбцам и производя соответствующие операции над строками, получим матрицу вида $(5)$, последняя строка которой $\left\|b_{1}^{(1)}(x) \ldots b_{t-1}^{(1)}(x) \Delta(x)\right\|$, где $b_{r}^{(1)}\left(\alpha_{1}\right)=\delta_{r 1}, b_{r}^{(1)}\left(\alpha_{t}\right)=0, r=1, \ldots, t-1$. Если $t>2$, то $\left\|b_{2}^{(1)}\left(\alpha_{2}\right) \ldots b_{t-1}^{(1)}\left(\alpha_{2}\right)\right\| \neq \overline{0}$, и мы можем считать $b_{2}^{(1)}\left(\alpha_{2}\right)=1$. Прибавляя некоторые кратные второго столбца ко всем остальным, кроме последнего, и производя соответствующие операции над строками, получим матрицу вида (5), последняя строка которой $\left\|b_{1}^{(2)}(x) \ldots b_{t-1}^{(2)}(x) \Delta(x)\right\|$, где $b_{r}^{(2)}\left(\alpha_{j}\right)=\delta_{r j}, b_{r}^{(2)}\left(\alpha_{t}\right)=0, r=1, \ldots, t-1$, $j=1,2$. Продолжая так и далее, в результате цепочки таких преобразований придем $\mathrm{K}$ матрице вида (5) с $t$-й строкой $\left\|c_{1}(x) \ldots c_{t-1}(x) \Delta(x)\right\|$, элементы которой удовлетворяют условиям (4).

ПРЕДЛОЖЕНИЕ 4. Если в матрицах

$$
C^{(m)}(x)=E_{n-t} \oplus\left\|\begin{array}{ccc|c}
\frac{E_{t-1}}{4(m)} & 0 \\
\hline c_{1}^{(m)}(x) & \ldots & c_{t-1}^{(m)}(x) & \Delta(x)
\end{array}\right\|, \quad m=1,2
$$


для некоторых характеристических корней $\alpha_{1}, \ldots, \alpha_{t}$ выполняются условия

$$
c_{i}^{(m)}\left(\alpha_{j}\right)=\delta_{i j}, \quad i, j=1, \ldots, t, \quad c_{t}^{(m)}(x)=1-\sum_{k=1}^{t-1} c_{k}^{(m)}(x)
$$

то условия $C^{(1)}(x) \sim C^{(2)}(x), M_{\bar{c}^{(1)}(x)}(\Delta) \stackrel{d}{\sim} M_{\bar{c}^{(2)}(x)}(\Delta)$, дде

$$
\bar{c}^{(m)}(x)=\left\|c_{1}^{(m)}(x) \quad \ldots \quad c_{t-1}^{(m)}(x) \quad c_{t}^{(m)}(x)\right\|
$$

әквивалентны.

ДокАЗАТЕЛЬСтво. Пусть $C^{(1)}(x) \sim C^{(2)}(x)$. Перейдем в очевидном равенстве

$$
S C^{(1)}(x)=C^{(2)}(x) R(x)
$$

где $S, R(x)$ - обратимые матрицы, к взаимньм матрицам. Из полученного равенства, обозначив через $\bar{r}_{n}(x)$ последнюю строку взаимной матрицы $R_{*}(x)$, имеем

$$
\begin{aligned}
& \|\underbrace{0 \ldots 0}_{n-t}-c_{1}^{(1)}(x) \quad \ldots \quad-c_{t-1}^{(1)}(x) \quad 1\| S_{*}
\end{aligned}
$$

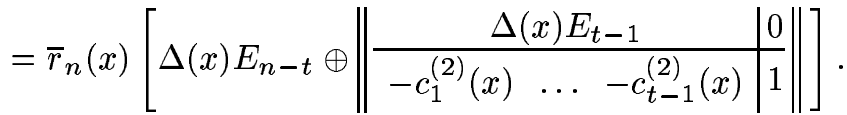

Переходя в последнем равенстве к значениям матриц на системе корней многочлена $\Delta(x)$ и учитьвая вид матрицы $C_{*}^{(2)}(x)$, получаем

$$
\begin{aligned}
& \left(M_{\left\|0 \ldots 0-c_{1}^{(1)}(x) \ldots-c_{t-1}^{(1)}(x) 1\right\|}(\Delta)\right) S_{*} \\
& \quad=\operatorname{diag}\left(r_{1}, \ldots, r_{p}\right) M_{\left\|0 \ldots 0-c_{1}^{(2)}(x) \ldots-c_{t-1}^{(2)}(x) 1\right\|}(\Delta)
\end{aligned}
$$

где $r_{i}$ - значения последнего элемента строки $\bar{r}_{n}(x)$ на системе корней многочлена $\Delta(x)$, $\left(r_{i} \neq 0\right)$. Из последнего можно получить

$$
\left(M_{\bar{c}^{(1)}(x)}(\Delta)\right) S^{\prime}=\operatorname{diag}\left(r_{1}, \ldots, r_{p}\right) M_{\bar{c}^{(2)}(x)}(\Delta)
$$

откуда, учитывая условия предложения, следует, что $S^{\prime}$ - диагональная неособенная матрица. Первая часть предложения доказана.

Обратно, пусть имеет место равенство

$$
\left(M_{\bar{c}^{(1)}(x)}(\Delta)\right) \operatorname{diag}\left(s_{1}, \ldots, s_{t}\right)=\operatorname{diag}\left(r_{1}, \ldots, r_{p}\right) M_{\bar{c}^{(2)}(x)}(\Delta),
$$

где все $s_{1}, \ldots, s_{t}, r_{1}, \ldots, r_{p}$ ненулевые. Отсюда можем записать

$$
\begin{aligned}
& \left(M_{\left\|0 \ldots 0-c_{1}^{(1)}(x) \ldots-c_{t-1}^{(1)}(x) 1\right\|}(\Delta)\right)\left(E_{n-t} \oplus\left\|c_{i j}\right\|\right) \\
& \quad=\operatorname{diag}\left(r_{1}, \ldots, r_{p}\right) M_{\left\|0 \ldots 0-c_{1}^{(2)}(x) \ldots-c_{t-1}^{(2)}(x) 1\right\|}(\Delta)
\end{aligned}
$$


где $\left\|c_{i j}\right\|$-матрица размеров $t \times t$,причем $c_{k k}=s_{k}, c_{k t}=s_{t}-s_{k}, k=1, \ldots, t-1, c_{t t}=s_{t}$ и все остальные элементы равны 0. По элементам матриц $\left\|c_{i j}\right\|, C^{(1)}(x)$ и $C^{(2)}(x)$ построим $(t \times t)$-матрицу $\left\|r_{i j}(x)\right\|$ следующим образом:

$$
\begin{gathered}
r_{t v}(x)=\frac{r_{t t}(x) c_{v}^{(2)}(x)-c_{v v} c_{v}^{(1)}(x)}{\Delta(x)} \\
r_{u v}(x)=c_{u v}+c_{u t} c_{v}^{(2)}(x), \quad r_{u t}(x)=c_{u t} \Delta(x), \quad u, v=1, \ldots, t-1, \\
r_{t t}(x)=c_{t t}-\sum_{h=1}^{t-1} c_{h t} c_{h}^{(1)}(x) .
\end{gathered}
$$

На основании (7) убеждаемся, что $\left\|r_{i j}(x)\right\| \in \mathbb{C}_{t}[x]$, и непосредственным подсчетом проверяем справедливость равенства

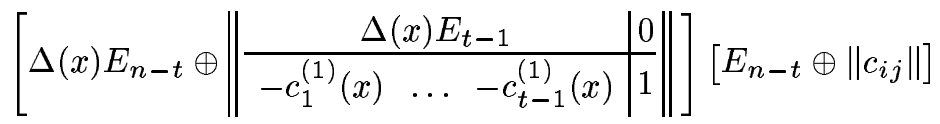

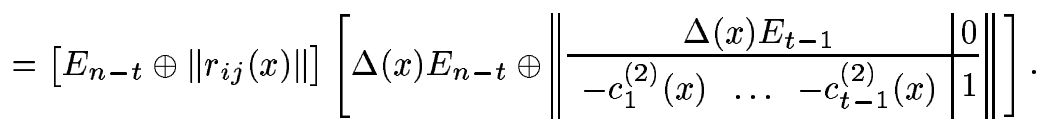

Отсюда следует равенство (6).

Пусть матрица $A(x)$ приведена к виду (3), описанному в предложении 3 . Тогда матрица $L=M_{\bar{c}(x)}(\Delta)$, где

$$
\bar{c}(x)=\left\|c_{1}(x) \quad \ldots \quad c_{t-1}(x) \quad c_{t}(x)\right\|, \quad c_{t}(x)=1-\sum_{p=1}^{t-1} c_{p}(x)
$$

перестановками строк приводится к виду

$$
L \rightarrow\left\|\begin{array}{c}
L_{1} \\
\cdots \\
L_{k}
\end{array}\right\|, \quad 1 \leqslant k \leqslant t
$$

где система строк каждой подматрищы $L_{i}$ связанная, а подматрищы $L_{j}$ и $L_{m}$ при $j \neq m$ несвязанные. Этим определяется некоторое разложение характеристического многочлена $\Delta(x)=\operatorname{det} A(x)$ :

$$
\Delta(x)=\prod_{i=1}^{k} \Delta_{i}(x)
$$

а именно: $M_{\bar{c}(x)}\left(\Delta_{i}\right)=L_{i}, i=1, \ldots, k$.

ПРЕДЛОЖЕНИЕ 5. Разложсение (8) классом полускалярно эквивалентных матрии $\{C A(x) Q(x)\}$ определяется однозначно. 
ДокАЗАТЕЛЬСТво. Пусть имеются полускалярно эквивалентные матрицы вида (3):

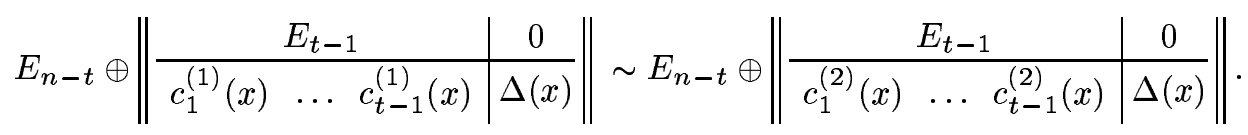

Тогда каждая из матриц $M_{\bar{c}^{(m)}(x)}(\Delta)(m=1,2)$, где

$$
\bar{c}^{(m)}(x)=\left\|c_{1}^{(m)}(x) \quad \ldots \quad c_{t-1}^{(m)}(x) \quad c_{t}^{(m)}(x)\right\|,
$$

содержит все строки единичной матрицы $E_{t}$. Для некоторого разложения $\Delta(x)=$ $\varphi_{1}(x) \varphi_{2}(x)$ построим матрицы

$$
M^{(m)}=\left\|\frac{M_{1}^{(m)}}{M_{2}^{(m)}}\right\|=\left\|\frac{M_{\bar{c}^{(m)}(x)}\left(\varphi_{1}\right)}{M_{\bar{c}^{(m)}(x)}\left(\varphi_{2}\right)}\right\|, \quad m=1,2 .
$$

Если матрицы $M_{1}^{(1)}, M_{2}^{(1)}$ несвязанные и $\operatorname{rank} M_{i}^{(1)}=n_{i}, i=1,2$, то $n_{1}+n_{2}=t=$ $\operatorname{rank} M^{(1)}$. Согласно предложению 4 из [2, 2, разд. II] $\operatorname{rank} M^{(2)}=t, \operatorname{rank} M_{i}^{(2)}=n_{i}$, $i=1,2$. Следовательно, из $t$ строк матрицы $E_{t}$, присутствующих в матрице $M^{(2)}$, ровно $n_{i}$ содержатся в матрице $M_{i}^{(2)}(i=1,2)$. Это значит, что матрицы $M_{1}^{(2)}, M_{2}^{(2)}$ несвязанные. Из приведенных рассуждений и вытекает заключение предложения.

ОПРЕДЕЛЕниЕ 5. Множители $\Delta_{i}(x)(i=1, \ldots, k)$ разложения (8) характеристического многочлена $\Delta(x)$ назьваются обобщенными әлементарными делителями (ОЭД) I рода матрицы $A(x)$.

Далее полезньп будет ввести понятие ступени матрицы.

ОПРедЕЛЕниЕ 6. Ступенью ненулевой матрицы $L$ (обозначение: st $L$ ) будем называть пару чисел $(p, q)$, где $p=(\operatorname{rank} L) / q, q$ - число ненулевых строк матрицы $L$, если на множестве таких пар определить отношение порядка: $(p, q)<\left(p^{\prime}, q^{\prime}\right)$ в случае $p<p^{\prime}$ или $p=p^{\prime}, q<q^{\prime}$.

Согласимся произвольную матрицу $B$, состоящую из некоторых строк матрицы $L$, называть строчной подматричей матрицы $L$. При этом $L$-номером строки $\bar{b}$ матрицы $B$ будем называть номер этой строки в матрице $L$.

Пусть $\Delta_{i}(x)$ - ОЭД І рода матрицы $A(x)$, приведенной к виду (3). Рассмотрим матрицу $L_{i}=M_{\bar{c}(x)}\left(\Delta_{i}\right)$. Нетрудно убедиться, что перестановками строк матрица $L_{i}$ приводится к виду

где

$$
L_{i} \rightarrow\left\|\begin{array}{c}
L_{i 1} \\
\ldots \\
L_{i t_{i}}
\end{array}\right\|=\widetilde{L}_{i}
$$

$$
\text { st }\left\|\begin{array}{c}
L_{i 1} \\
\cdots \\
L_{i, j-1} \\
L_{i j}
\end{array}\right\| \leqslant \mathrm{st}\left\|\begin{array}{c}
L_{i 1} \\
\cdots \\
L_{i, j-1} \\
H_{i j}
\end{array}\right\|, \quad j=1, \ldots, t_{i},
$$

$H_{i j}$ - произвольная строчная подматрица матрищы, полученной из $L_{i}$ вычеркиванием подматриц $L_{i 1}, \ldots, L_{i, j-1}$, причем в случае равенства ступеней указанных матрищ 
минимальный $L_{i}$-номер строк подматрицы $L_{i j}$ меньше минимального $L_{i}$-номера строк подматрищы $H_{i j}$. Очевидно, с точностью до размешения строк в пределах каждой из подматрищ $L_{i j}$ матрища $\widetilde{L}_{i}$ определяется однозначно. Ей соответствует некоторое разложение ОЭД I рода $\Delta_{i}(x)$ :

$$
\Delta_{i}(x)=\prod_{j=1}^{t_{i}} \Delta_{i j}(x)
$$

так что $L_{i j}=M_{\bar{c}(x)}\left(\Delta_{i j}\right), j=1, \ldots, t_{i}$. Таким образом, от разложения $(8)$ характеристического многочлена $\Delta(x)$ матрицы $A(x)$ на ОЭД I рода приходим к разложению

$$
\Delta(x)=\prod_{i=1}^{k} \prod_{j=1}^{t_{i}} \Delta_{i j}(x)
$$

ПРЕДЛОЖЕНИЕ 6. Разложсение (9) классом полускалярно эквивалентных матрии $\{C A(x) Q(x)\}$ определяется однозначно.

Доказательство легко провести, используя предложение 4 из $[2, \S 2$, разд. II] и определение 6.

ОПРЕДЕЛЕНИЕ 7. Множители $\Delta_{i j}(x)\left(i=1, \ldots, k, j=1, \ldots, t_{i}\right)$ разложения (9) характеристического многочлена $\Delta(x)$ назьваются обобщенными әлементарными делителями II рода матрищы $A(x)$.

ОПРЕдЕЛЕниЕ 8. Матрица $F(x)$ без кратных корней характеристического многочлена $\Delta(x)$ с ОЭД I рода $\Delta_{i}(x)(i=1, \ldots, k)$ и ОЭД II рода $\Delta_{i j}(x)(i=1, \ldots, k$, $\left.j=1, \ldots, t_{i}\right)$ называется канонической в классе полускалярно эквивалентных матрищ, если она имеет вид

$$
F(x)=\bigoplus_{i=1}^{k} F_{i}(x) \oplus E_{n-t}
$$

где

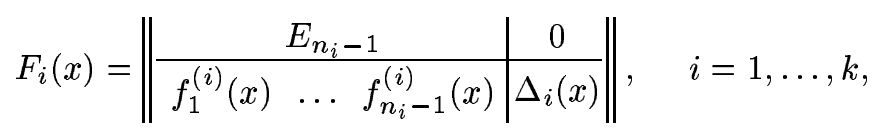

$\operatorname{deg} f_{m}^{(i)}<\operatorname{deg} \Delta_{i}, \quad m=1, \ldots, n_{i}-1, \quad n_{i}=\operatorname{rank} M_{F_{*}(x)}\left(\Delta_{i}\right), \quad t=\operatorname{rank} M_{F_{*}(x)}(\Delta)$, и матрища

$$
\begin{gathered}
\left\|\begin{array}{c}
M_{\bar{f}^{(i)}(x)}\left(\Delta_{i 1}\right) \\
\cdots \\
M_{\bar{f}^{(i)}(x)}\left(\Delta_{i t_{i}}\right)
\end{array}\right\|, \quad \bar{f}^{(i)}(x)=\| f_{1}^{(i)}(x) \\
f_{n_{i}}^{(i)}(x)=1
\end{gathered}
$$

в которой первые $n_{i}$ линейно независимьх строк образуют единичную матрицу $F_{n_{i}}$, является канонической в классе псевдостохастических диагонально эквивалентных матриц. 
Tеорема 1. В классе полускалярно әквивалентных матрии без кратных характеристических корней существует, причем единственная с точностью до порядка прямых слагаемых, каноническая матрица.

ДокАЗАтельСтво. Пусть $\Delta_{i}(x), \Delta_{i j}(x)\left(i=1, \ldots, k, j=1, \ldots, t_{i}\right)$ - соответственно ОЭД I и II рода матрицы $A(x)$, которая согласно предложению 3 полускалярно эквивалентна матрице (3). Построим матрицу $L_{i}=M_{\bar{c}(x)}\left(\Delta_{i 1} \cdots \Delta_{i t_{i}}\right)(i=1, \ldots, k)$, где $\bar{c}(x)=\left\|c_{1}(x) \ldots c_{t-1}(x) c_{t}(x)\right\|$. Обозначим через $n_{i}$ число ее ненулевых столбцов, а через $\bar{c}^{(i)}(x)$ строку, состоящую из тех элементов строки $\bar{c}(x)$, которые соответствуют этим $n_{i}$ ненулевым столбцам матрицы $L_{i}(i=1, \ldots, k)$. Поскольку $\operatorname{rank}\left\|L_{1}^{T} \ldots L_{k}^{T}\right\|^{T}$ $=t$ и матрицы $L_{i}(i=1, \ldots, k)$ попарно несвязанные, то $n_{1}+\cdots+n_{k}=t$ и строки $\left\|\bar{c}^{(1)}(x) \ldots \bar{c}^{(k)}(x)\right\|, \bar{c}(x)$ различаются, возможно, лишь размещением элементов. Приведем строки $\bar{c}^{(i)}(x)(i=1, \ldots, k)$ по модулю $\Delta_{i}(x)$ :

$$
\bar{c}^{(i)}(x) \equiv \bar{d}^{(i)}(x)\left(\bmod \Delta_{i}(x)\right), \quad \bar{d}^{(i)}(x)=\left\|\bar{d}_{1}^{(i)}(x) \quad \ldots \quad \bar{d}_{n_{i}-1}^{(i)}(x) \bar{d}_{n_{i}}^{(i)}(x)\right\|,
$$

и построим матрицу

$$
D(x)=\bigoplus_{i=1}^{k}\left\|\begin{array}{c|c}
E_{n_{i}-1} & 0 \\
\hline \bar{d}_{0}^{(i)}(x) & \Delta_{i}(x)
\end{array}\right\| \oplus E_{n-t},
$$

где $\bar{d}_{0}^{(i)}(x)=\left\|\bar{d}_{1}^{(i)}(x) \ldots \bar{d}_{n_{i}-1}^{(i)}(x)\right\|$. Покажем, что $C(x) \sim D(x)$. Доказательство этого факта достаточно провести для $k=2$. Так как НОД $\left(\Delta_{1}(x), \Delta_{2}(x)\right)=1$, то существуют $u_{1}(x), u_{2}(x) \in \mathbb{C}[x]$ такие, что $u_{1}(x) \Delta_{1}(x)+u_{2}(x) \Delta_{2}(x)=1, \operatorname{deg} u_{1}<\operatorname{deg} \Delta_{2}$, $\operatorname{deg} u_{2}<\operatorname{deg} \Delta_{1}$. Следовательно,

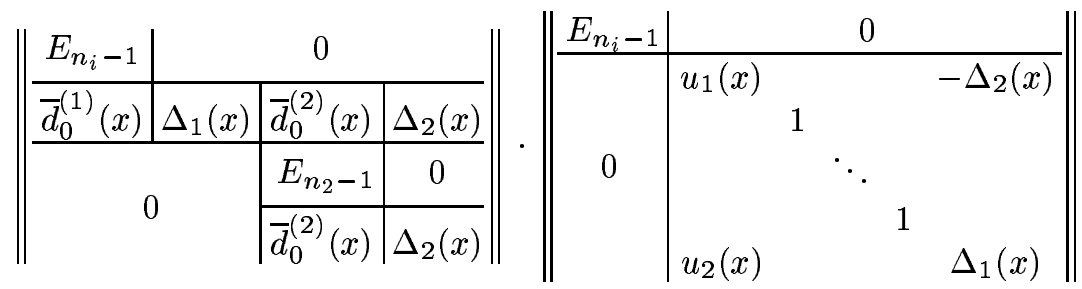

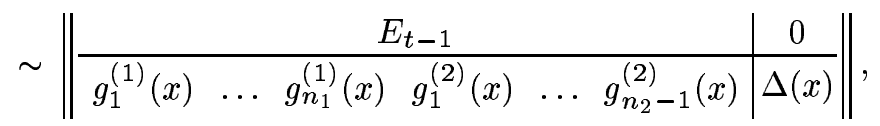

где

$$
\begin{aligned}
& g_{p}^{(1)}(x) \equiv u_{2}(x) \Delta_{2}(x) d_{p}^{(1)}(x)(\bmod \Delta(x)), \quad p=1, \ldots, n_{1}, \\
& g_{m}^{(2)}(x) \equiv u_{1}(x) \Delta_{1}(x) d_{m}^{(2)}(x)(\bmod \Delta(x)), \quad m=1, \ldots, n_{2}-1 .
\end{aligned}
$$

Легко видеть, что строки

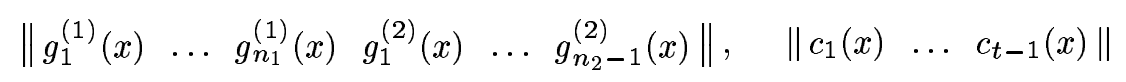

имеют степени меньше чем $\operatorname{deg} \Delta$ и принимают одинаковые значения на системе корней многочлена $\Delta(x)$. Поэтому они равны и нужное доказано. Учитьвая предложение 3 , можем считать, что первые $n_{i}$ линейно независимых строк матрицы

$$
\widetilde{L}_{i}=M_{\bar{d}^{(i)}(x)}\left(\Delta_{i 1} \cdots \Delta_{i t_{i}}\right), \quad i=1, \ldots, k,
$$


образуют единичную матрицу $E_{n_{i}}$. Далее, для каждой из матриц $\widetilde{L}_{i}$ в классе псевдостохастических диагонально эквивалентных матриц находим каноническую $N_{i}$ и по интерполяционньм формулам строим строки $\bar{f}^{(i)}(x)=\left\|\bar{f}_{1}^{(i)}(x) \ldots \bar{f}_{n_{i}}^{(i)}(x)\right\|$ такие, что $N_{i}=M_{\bar{f}_{(x)}^{(i)}}\left(\Delta_{i 1} \cdots \Delta_{i t_{i}}\right)$. Затем по элементам $\bar{f}_{m}^{(i)}(x)$ строим матрищу вида $(10)$, имеющую каноническую форму. Последняя согласно предложению 4 полускалярно эквивалентна матрице $D(x)$.

Единственность следует из предложений 2, 4 .

ОПРЕДЕЛЕНИЕ 9 . Строки $\left\|\bar{f}_{1}^{(i)}(x) \ldots \bar{f}_{n_{i}-1}^{(i)}(x) \bar{f}_{n_{i}}^{(i)}(x)\right\|(i=1, \ldots, k)$, где

$$
f_{n_{i}}^{(i)}(x)=1-\sum_{m=1}^{n_{i}-1} f_{m}^{(i)}(x)
$$

и все $\bar{f}_{m}^{(i)}(x)$ взяты из канонического вида (10) матрицы $F(x)$, называются инвариантными многочленными строками матрицы $A(x) \sim F(x)$.

4. Подобие матричных многочленов. Рассмотрим набор из $s$ квадратных матриц над $\mathbb{C}$ порядка $n$ :

$$
\mathscr{A}=\left(A_{1}, \ldots, A_{s}\right) .
$$

Сопоставим ему матричный многочлен

$$
A(x)=E_{n} x^{s}-A_{1} x^{s-1}-\cdots-A_{s} .
$$

Будем предполагать, что все корни характеристического многочлена $\Delta(x)=\operatorname{det} A(x)$ просты. Цель этого пункта - построение канонической формы набора числовых матриц (11) в классе подобных наборов $\left\{\left(P A_{1} P^{-1}, \ldots, P A_{s} P^{-1}\right)\right\}, P \in \mathrm{GL}_{n}(\mathbb{C})$ или, что то же самое, построение канонической формы матричного многочлена (12) в классе подобных многочленов $\left\{P A(x) P^{-1}\right\}$.

ОПРЕДЕЛЕНИЕ 10. Матричный многочлен $I(x)=E_{n} x^{s}-I_{1} x^{s-1}-\cdots-I_{s}$ без кратньх характеристических корней назьвается канонической клеткой, если он имеет лишь один ОЭД І рода, соответственно одну инвариантную многочленную строку $\bar{f}(x)$, и ОЭД II рода $\Delta_{11}(x), \ldots, \Delta_{1 t_{1}}(x)$, с которыми он связан следующим образом:

$$
\begin{aligned}
\left\|\begin{array}{c}
I_{s} \\
\cdots \\
I_{1}
\end{array}\right\|= & \left\|M_{x^{0} \bar{f}(x)}\left(\Delta_{11} \cdots \Delta_{1 t_{1}}\right)|\ldots| M_{x^{s-1} \bar{f}(x)}\left(\Delta_{11} \cdots \Delta_{1 t_{1}}\right)\right\|^{-1} \\
& \times M_{x^{s} \bar{f}(x)}\left(\Delta_{11} \cdots \Delta_{1 t_{1}}\right) .
\end{aligned}
$$

Напомним, что матричньй многочлен назьвается приводимым, если преобразованием подобия он приводится к квазитреугольному виду. В противном случае говорят, что многочлен неприводимьй.

ПРЕДЛОЖЕНИЕ 7. Каноническая клетка $\kappa$ квазидиагональному виду не приводится. Она либо является неприводимым матричным многочленом, если число $t_{1}$ ОЭД II рода равно 1 , либо имеет квазитреугольный вид с $t_{1}$ неприводимыми диагональными блоками, если $t_{1}>1$. В последнем случае ОЭД II рода являются характеристическими многочленами диагональных блоков квазитреугольного вида канонической клетки. 
ДокАЗАтЕльство. Пусть дана каноническая клетка $I(x)$ степени $s$ порядка $n$ и $\bar{f}(x)$ - ее инвариантная многочленная строка. Предположим сначала вопреки заключению предложения, что преобразованием подобия многочлен $I(x)$ приводится к квазидиагональному виду с двумя диагональньми блоками порядков $p_{1}, p_{2}$ и характеристическими многочленами $\Delta_{1}(x), \Delta_{2}(x)$ соответственно. Тогда $\operatorname{deg} \Delta_{i}=s p_{i}$ и, применяя предложение 4 из $[2, \S 2$, разд. II] и теорему 5 из [13], получаем

$$
\operatorname{rank} M_{\bar{f}(x)}\left(\Delta_{i}\right)=\operatorname{rank} M_{I_{*}(x)}\left(\Delta_{i}\right)=p_{i}, \quad i=1,2 .
$$

Это значит, что система строк матрицы $M_{\bar{f}(x)}\left(\Delta_{1} \Delta_{2}\right)$ несвязанная, что невозможно, ибо $I(x)$ имеет лиш один ОЭД I рода.

Допустим теперь, что многочлен $I(x)$ с одним лишь ОЭД II рода $\left(t_{1}=1\right)$ преобразованием подобия приводится к квазитреугольному виду с двумя диагональными блоками порядков $p_{1}, p_{2}$ и характеристическими многочленами $\Delta_{1}(x), \Delta_{2}(x)$ соответственно. Тогда $\operatorname{deg} \Delta_{i}=s p_{i}, i=1,2$, и на основании теоремы I из [2, 3, разд. V] имеет место одно из равенств (14) (для $i=1$ или $i=2$ ). Поэтому для одной из строчных подматриц $M_{\bar{f}(x)}\left(\Delta_{i}\right)(i=1,2)$ матрицы $M_{\bar{f}(x)}\left(\Delta_{1} \Delta_{2}\right)$ вьполняется условие

$$
\text { st } M_{\bar{f}(x)}\left(\Delta_{i}\right)=\left(\frac{1}{s}, s p_{i}\right)<\left(\frac{1}{s}, s n\right)=\operatorname{st} M_{\bar{f}(x)}\left(\Delta_{1} \Delta_{2}\right) \text {, }
$$

что влечет за собой существование по крайней мере двух ОЭД II рода.

Оставшуюся часть предложения достаточно доказать для $t_{1}=2$. Пусть $\Delta_{11}(x)$, $\Delta_{12}(x)$ - ОЭД II рода матрицы $I(x), \operatorname{deg} \Delta_{11}=d, d=s p+h, 0 \leqslant h<s$. Поскольку st $M_{\bar{f}(x)}\left(\Delta_{11}\right)<\operatorname{st} M_{\bar{f}(x)}\left(\Delta_{11} \Delta_{12}\right)$, то

$$
\frac{1}{d} \operatorname{rank} M_{\bar{f}(x)}\left(\Delta_{11}\right) \leqslant \frac{1}{s n} \operatorname{rank} M_{\bar{f}(x)}\left(\Delta_{11} \Delta_{12}\right) .
$$

Следовательно,

$$
\operatorname{rank} M_{\bar{f}(x)}\left(\Delta_{11}\right) \leqslant \operatorname{rank} M_{\bar{f}(x)}\left(\Delta_{11} \Delta_{12}\right) .
$$

Если $\operatorname{rank} M_{\bar{f}(x)}\left(\Delta_{11}\right)=p_{1}$, то согласно определениям 8,9 матрища $M_{\bar{f}(x)}\left(\Delta_{11}\right)$ содержит $p_{1}$ первых строк единичной матрицы $E_{n}$; следовательно, ее последние $n-p_{1}$ столбцов нулевые. Из теоремы 3 из $\left[2, \S 2\right.$, разд. III] имеем, что $\operatorname{rank} M_{\bar{f}(x)}\left(\Delta_{11} \Delta_{12}\right)=n$. Поэтому из неравенства $(15)$ получаем $p_{1} /(s p+h)<1 / s$, откуда $p_{1}<p$. Снова обращаясь к теореме 3 из $[2, \S 2$, разд. III], имеем

$$
\operatorname{rank}\left\|M_{x^{0} \bar{f}(x)}\left(\Delta_{11} \Delta_{12}\right)|\ldots| M_{x^{s-1} \bar{f}(x)}\left(\Delta_{11} \Delta_{12}\right)\right\|=s n .
$$

Таким образом, $p=p, h=0$ и на самом деле $d=s p$. Из вида матриц

$$
\left\|M_{x^{0} \bar{f}(x)}\left(\Delta_{11} \Delta_{12}\right)|\ldots| M_{x^{s-1} \bar{f}(x)}\left(\Delta_{11} \Delta_{12}\right)\right\|^{-1}, \quad M_{x^{s} \bar{f}(x)}\left(\Delta_{11} \Delta_{12}\right)
$$

и формулы (13) вытекает, что коэффициенты $I_{1}, \ldots, I_{s}$ многочлена $I(x)$ имеют одинаковую квазитреугольную структуру, поэтому

$$
I(x)=\left\|\begin{array}{cc}
D_{1}(x) & 0 \\
* & D_{2}(x)
\end{array}\right\|
$$


где $D_{1}(x)-(p \times p)$-матрица, $\operatorname{det} D_{i}(x)=\Delta_{1 i}(x), i=1,2$. Если один из диагональных блоков матрицы $I(x)$ приводимьй, то, принимая во внимание теорему 1 из [2, 3 , разд. V], либо матрища $M_{\bar{f}(x)}\left(\Delta_{11}\right)$ имеет собственную строчную подматрицу ступени меньше чем st $M_{\bar{f}(x)}\left(\Delta_{11}\right)$, либо матрица $M_{\bar{f}(x)}\left(\Delta_{12}\right)$ имеет собственную строчную подматрицу такую, что в совокупности с матрицей $M_{\bar{f}(x)}\left(\Delta_{11}\right)$ ее ступень меньше чем st $M_{\bar{f}(x)}\left(\Delta_{11} \Delta_{12}\right)$. В обоих случаях получаем противоречие с условием, что $I(x)$ имеет лишь два ОЭД II рода.

ОПРЕДЕЛЕниЕ 11. Матричньй многочлен без кратных характеристических корней назьвается каноническим, если он является прямой суммой канонических клеток.

Теорема 2. В классе подобных матричных многочленов существует, причем единственный с точностью до перестановки канонических клеток, канонический матричный многочлен.

ДокАЗАТЕЛЬСтвО. Сушествование. Пусть $\Delta_{i}(x)$ и $\Delta_{i j}(x)$ - ОЭД I и II рода соответственно матричного многочлена (12),

$$
\bar{f}^{(i)}(x)=\left\|\bar{f}_{1}^{(i)}(x) \quad \ldots \quad \bar{f}_{n_{i}-1}^{(i)}(x) \bar{f}_{n_{i}}^{(i)}(x)\right\|, \quad i=1, \ldots, k, \quad j=1, \ldots, t_{i},
$$

- его инвариантные многочленные строки $\left(n_{1}+\cdots+n_{k}=n\right)$. Построим матрицу

$$
F(x)=\bigoplus_{i=1}^{k} F_{i}(x)
$$

где

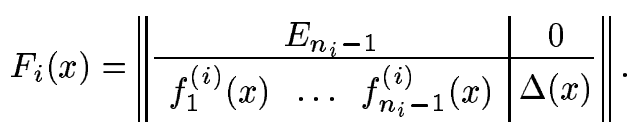

Очевидно, $F_{i}(x)$ - каноническая в классе полускалярно эквивалентных матриц, причем $\Delta_{i}(x), \Delta_{i j}(x)\left(j=1, \ldots, t_{i}\right)$ и $\bar{f}^{(i)}(x)$ - ее ОЭД I, II рода и инвариантная многочленная строка соответственно. Поэтому матричньй многочлен

$$
I^{(i)}(x)=E_{n_{i}} x^{s}-I_{1}^{(i)} x^{s-1}-\cdots-I_{s}^{(i)},
$$

коэффищиенты которого определяются по формуле

$$
\begin{aligned}
\left\|\begin{array}{c}
I_{s}^{(i)} \\
\ddot{I_{1}^{(i)}}
\end{array}\right\|= & \left\|M_{x^{0} \bar{f}^{(i)}(x)}\left(\Delta_{i 1} \cdots \Delta_{i t_{i}}\right)|\ldots| M_{x^{s-1} \bar{f}^{(i)}(x)}\left(\Delta_{i 1} \cdots \Delta_{i t_{i}}\right)\right\|^{-1} \\
& \times M_{x^{s} \bar{f}^{(i)}(x)}\left(\Delta_{i 1} \cdots \Delta_{i t_{i}}\right),
\end{aligned}
$$

является канонической клеткой, а матричньй многочлен

$$
I(x)=\bigoplus_{i=1}^{k} I^{(i)}(x)
$$

- каноническим матричным многочленом в классе подобных. Очевидно, $A(x) \sim F(x)$, и согласно теореме 2 из [2, 3 , разд. III] $F(x) \sim I(x)$. Следовательно, $A(x) \sim I(x)$ и в силу [9] $A(x)=P I(x) P^{-1}$. 
Единственность канонического матричного многочлена в классе подобных следует из единственности канонической формы в классе полускалярно эквивалентных матриц и однозначности определения формулой (13) коэффициента канонической клетки. Теорема доказана.

Таким образом, по набору числовых матриц $\mathscr{A}$ и соответствующему ему матричному многочлену $A(x)$ мы построили канонический матричньй многочлен $I(x)$, подобный $A(x)$. Набор коэффициентов многочлена $I(x)$ - подобньй набору $\mathscr{A}$. Он является каноническим в классе подобных, поскольку имеет наиболее простую форму и определяется однозначно.

В заключение отметим, что ОЭД I рода многочленной матрицы являются характеристическими многочленами канонических клеток ее канонической формы, а ОЭД II рода - характеристическими многочленами неприводимых диагональных блоков квазитреугольного вида этих клеток. Заметим также, что в случае, когда набор состоит из одной лишь матрицы, построенная здесь каноническая форма является диагональной, следовательно, совпадает с формой Жордана этой матрицы.

\section{СПИСОК ЦИТИРОВАННОЙ ЛИТЕРАТУРЫ}

[1] Кравчук М.П. Про квадратичні форми та лінійні перетворення // Тр. фіз.-мат. відділу УАН. 1924. Т. 1. №3. С. 1-91.

[2] Казімірський П. С. Розклад матричных многочленів на множники. Киів: Наукова думка, 1981.

[3] Gohberg I, Lancaster P., Rodman L. Matrix Polynomials. New York: Acad. Press, 1982.

[4] Friedland S. Simultaneous similarity of matrices // Adv. Math. 1983. V. 50. P. 189-265.

[5] Baratchart L. Un theoreme de factrorisation et son application à la representation des systèmes cyclique causaux // C. R. Acad. Sci. Paris. Sér. I. Math. 1982. V. 295. № 3. P. 223-226.

[6] Гантмахер Ф.Р. Теория матриц. М.: Наука, 1966.

[7] Мальцев А.И. Основы линейной алгебры. М.: Наука, 1970.

[8] Казимирский П. С., Петричкович В.М. К выделению из полиномиальной матрицы линейного множителя с заданной системой инвариантных многочленов // III Всесоюзный симпозиум по теории колец, алгебр и модулей. Тезисы докл. Тарту, 1976. С. 19.

[9] Казімірьский П. С., Петричкович В. М. Про еквівалентність поліноміальних матриць // Теоретичні та прикладні питання алгебри і дифференціальних рівнянь. Киів: Наукова думка, 1977. С. 61-66.

[10] Казимирский П. С., Мельник О. М. Подобие и строение унитальных матричных квадратных трехчленов с попарно различными характеристическими корнями // Зап. научн. семинаров ЛОМИ. 1989. Т. 175. С. 63-68.

[11] Казимирский П. С., Билонога Д. М. Полускалярная эквивалентность многочленньх матриц с взаимно простыми элементарными делителями // Докл. АН УССР. Сер. А. 1990. T. 4. C. $8-9$.

[12] Шаваровский Б. З. Полускалярная эквивалентность полиномиальных матриц с попарно различными корнями их характеристического многочлена // Матем. методы и физ.-мех. поля. Вып. 19. Киев: Наукова думка, 1984. С. 33-37.

[13] Шаваровский Б. З. О приводимости матричных многочленов к блочно-треугольному и блочно-диагональному видам // Материалы 10-й конференции молодых ученых Института прикладных проблем механики и математики АН УССР. Ч. 2. Львов, 1984. С. 225-228. Деп. ВИНИТИ № 7197-84.

Институт прикладных проблем механики и математики

Поступило им. Я. С. Подстригача НАН Украины, г. Львов 29.02 .96

Исправленный вариант 20.04 .98 